\title{
Intravesical administration of small interfering RNA targeting PLK-1 successfully prevents the growth of bladder cancer
}

\author{
Masaki Nogawa, ${ }^{1}$ Takeshi Yuasa, ${ }^{1}$ Shinya Kimura, ${ }^{1}$ Motoyoshi Tanaka, ${ }^{2}$ Junya Kuroda, ${ }^{1}$ \\ Kiyoshi Sato,, ${ }^{1}$ Asumi Yokota, ${ }^{1}$ Hidekazu Segawa, ${ }^{1}$ Yoshinobu Toda, ${ }^{3}$ Susumu Kageyama, ${ }^{4}$ \\ Tatsuhiro Yoshiki, ${ }^{4}$ Yusaku Okada, ${ }^{4}$ and Taira Maekawa1 \\ 1Department of Transfusion Medicine and Cell Therapy, Kyoto University Hospital, Kyoto, Japan. ${ }^{2}$ Department of Urology, Nara Medical University, \\ Nara, Japan. ${ }^{3}$ Center for Anatomical Studies, Kyoto University Graduate School of Medicine, Kyoto, Japan. ${ }^{4}$ Department of Urology, \\ Shiga University of Medical Science, Shiga, Japan.
}

\begin{abstract}
The mainstay in the management of invasive bladder cancer continues to be radical cystectomy. With regard to improvement of quality of life, however, therapies that preserve the bladder are desirable. We investigated the use of intravesical PLK-1 small interfering RNA (siRNA) against bladder cancer. Patients with bladder cancers expressing high levels of PLK-1 have a poor prognosis compared with patients with low expression. Using siRNA/cationic liposomes, the expression of endogenous PLK-1 could be suppressed in bladder cancer cells in a time- and dose-dependent manner. As a consequence, PLK-1 functions were disrupted. Inhibition of bipolar spindle formation, accumulation of cyclin B1, reduced cell proliferation, and induction of apoptosis were observed. In order to determine the efficacy of the siRNA/liposomes in vivo, we established an orthotopic mouse model using a LUC-labeled bladder cancer cell line, UM-UC-3 ${ }^{\text {LUC }}$. PLK-1 siRNA was successfully transfected into the cells, reduced PLK-1 expression, and prevented the growth of bladder cancer in this mouse model. This is the first demonstration, to our knowledge, of inhibition of cancer growth in the murine bladder by intravesical siRNA/cationic liposomes. We believe intravesical siRNA instillation against bladder cancer will be useful as a therapeutic tool.
\end{abstract}

\section{Introduction}

Approximately $70 \%$ of bladder cancers are at a superficial stage at initial diagnosis $(1,2)$. These lesions are usually managed with transurethral resection, followed by intravesical administration of agents including mitomycin $\mathrm{C}$, adriamycin, and bacille Calmette-Guérin (BCG). Although these intravesical agents can prolong the length of progression-free survival after the cancers have been resected, long-term follow up has demonstrated that they infrequently cured patients of bladder cancer $(1,2)$. Half of superficial lesions will recur, and as many as $10-30 \%$ will progress to a higher grade and/or stage and form local invasive cancers (1-3). The standard treatment for local invasive cancer is radical cystectomy, which may result in loss of urinary and sexual functions. Consequently, the current treatment often adversely affects the quality of life of these patients. Therefore, a novel therapeutic approach against superficial cancer has been eagerly desired.

RNA interference is a newly discovered cellular pathway for silencing genes in a sequence-specific manner at the mRNA level by the introduction of the cognate double-stranded small interfering RNA (siRNA) and has recently been experimentally introduced into cancer therapy, primarily in vitro $(4,5)$. In vivo, however, the therapeutic application of siRNAs is largely dependent on the development of a drug delivery system (DDS) that efficiently

Nonstandard abbreviations used: BCG, bacille Calmette-Guérin; DDS, drug delivery system; IVIS, in vivo imaging system; MTT, 3-(4,5-dimethylthiazol-2-yl)-2,5-diphenyltetrazolium bromide; PLK-1, polo-like kinase-1; siRNA, small interfering RNA. Conflict of interest: The authors have declared that no conflict of interest exists.

Citation for this article: J. Clin. Invest. 115:978-985 (2005)

doi:10.1172/JCI200523043. delivers the siRNAs to target cells, and, therefore, siRNA has not yet entered clinical trials.

Polo-like kinase-1 (PLK-1) is one of the most important regulators of mitotic progression in mammalian cells (6). Moreover, elevation of PLK-1 expression is positively correlated with a broad range of human tumors (7-10), and depletion of PLK-1 by siRNA dramatically inhibited cell growth and induced apoptosis in vitro $(11,12)$. In this study, we investigated the use of intravesical PLK-1 siRNA as a possible therapeutic tool against bladder cancer. We used cationic liposomes as the in vivo carrier. Recently, a complex of polyinosinic-polycytidylic acid and cationic liposomes successfully inhibited the growth of, and induced apoptosis in, a mouse liver metastasis (13). This simple vector scheme is attractive. We predicted that local administration, such as intravesical instillation of the complex of siRNA and liposomes, can provide a relatively high concentration of siRNA to cancer cells. PLK-1 siRNA was successfully transfected into the cells, reduced PLK-1 expression, and prevented the growth of bladder cancer in an orthotopic murine model. This is the first demonstration, to our knowledge, of inhibition of cancer growth in the murine bladder by intravesical siRNA/cationic liposomes.

\section{Results}

Relationship between the expression of PLK-1 and clinicopathological features in radical-cystectomy patients. We initially tested whether immunohistochemical levels of PLK-1 expression were related to various clinicopathological features and survival rates in patients with bladder cancer. The histologically high-grade, deeply invasive, lymphatic-invasive, and venous-invasive bladder cancers demonstrated significantly higher PLK-1 expression than did the lowgrade, superficial, non-lymphatic-invasive, and non-venous- 
Table 1

Relationship between PLK-1 expression level and clinicopathological features of human bladder cancers

\begin{tabular}{|c|c|c|c|c|c|}
\hline & & Total & High & Low & $P$ value \\
\hline Age & Mean (SD) & $64.6(10.0)$ & $65.8(9.27)$ & $63.4(10.6)$ & 0.347 \\
\hline \multirow[t]{2}{*}{ Sex } & Male & 48 & 22 & 26 & 0.297 \\
\hline & Female & 10 & 7 & 3 & \\
\hline \multirow[t]{2}{*}{ Configuration } & Papillary & 16 & 6 & 10 & 0.189 \\
\hline & Nonpapillary & 42 & 23 & 19 & \\
\hline \multirow[t]{2}{*}{ Number of tumors } & Solitary & 30 & 18 & 12 & 0.44 \\
\hline & Multiple & 28 & 11 & 17 & \\
\hline \multirow[t]{2}{*}{ Grade } & Well/Mod & 18 & 2 & 16 & $<0.001$ \\
\hline & Poor & 40 & 27 & 13 & \\
\hline \multirow[t]{2}{*}{ Depth of invasion } & Superficial & 21 & 3 & 18 & $<0.001$ \\
\hline & Invasive & 37 & 26 & 11 & \\
\hline \multirow[t]{2}{*}{ Lymph node involvement } & Negative & 51 & 24 & 27 & 0.420 \\
\hline & Positive & 7 & 5 & 2 & \\
\hline \multirow[t]{3}{*}{ Lymphatic invasion } & Negative & 15 & 0 & 15 & $<0.001$ \\
\hline & Positive & 31 & 21 & 10 & \\
\hline & Unknown & 12 & 8 & 4 & \\
\hline \multirow[t]{3}{*}{ Venous invasion } & Negative & 39 & 15 & 24 & $<0.05$ \\
\hline & Positive & 8 & 7 & 1 & \\
\hline & Unknown & 11 & 7 & 4 & \\
\hline Follow-up (month) & Mean & $68.7(48.0)$ & $63.5(53.8)$ & $73.7(41.8)$ & 0.469 \\
\hline
\end{tabular}

Mod, moderate.

invasive cancers (Figure 1A and Table 1). Moreover, the patients with high PLK-1 expression had a significantly worse prognosis than patients with low PLK-1 expression (Figure 1B; $P<0.05$ ).

We then investigated PLK-1 expression in the tissues of bladder cancer and normal bladder epithelium by Western blotting (Figure 1C). Cancerous tissues had a more abundant expression of PLK-1 than normal epithelium. Moreover, abundant expression of PLK-1 was demonstrated in all but 1 (RT4) of the bladder cancer cell lines examined (Figure 1D). These observations suggested that altered PLK-1 expression might be instrumental in cancer initiation or progression in bladder cancers. Thus, PLK-1 was indicated as an attractive therapeutic molecular target for bladder cancer. We next tested the effect of reducing expression of PLK-1 in bladder cancer cells using siRNAs in vitro.

Inhibition of PLK-1 expression by siRNAs. Four PLK-1 siRNAs were introduced into cells using cationic liposomes. We examined the ability of siRNAs to reduce the endogenous level of PLK-1 protein in HeLa, HT1376, and MBT2 cancer cell lines. Transfection of these cell lines with PLK-1 siRNAs alone demonstrated no effect (data not shown). Transfection with PLK-1 siRNAs and cationic liposomes at a concentration $50 \mathrm{nM}$ did not reduce PLK-1 at 6 hours after transfection but led to a marked loss of PLK-1 at 24 hours after transfection (Figure 2A). Among the siRNAs we tested, PLK-1 siRNA 1412, which completely matched the mRNA sequence of human, mouse, and rat PLK-1, possessed the strongest inhibitory effect against PLK-1 expression.

PLK-1 siRNA 1412 inhibited PLK-1 expression in a time- and dose-dependent manner (Figure 2, B and C). We used PLK-1 siRNA 1412 with cationic liposomes in subsequent experiments. PLK-1 is known to play important roles in mitosis (6). Depletion of PLK-1 induces M-phase arrest and results in accumulation of cyclin B1. In order to examine the inhibitory effect of siRNA 1412 against PLK-1 functions, we investigated cyclin B1 expression in bladder cancer cells after siRNA treatment. The expression of cyclin B1 was dose-dependently increased by siRNA 1412 (Figure 2C).

We performed anti- $\beta$-tubulin and anti- $\gamma$-tubulin immunocytochemistry and DNA staining of siRNA-treated bladder cancer cells to observe spindle formation. One of the well-known features of PLK-1 is that reduced PLK-1 activity results in aberrant spindle-pole formation, which is reflected in the name for this kinase family (6). In control cells, normal M-phase bipolar spindles could be observed by $\beta$ - and $\gamma$-tubulin staining, and chromosomal DNA was aligned at the center of the cells as visualized by Hoechst 33342 staining. In PLK-1 siRNA-treated bladder cancer cells, the numbers of $\mathrm{M}$-phase rounded cells were increased, and bipolar spindle formations were dramatically reduced. DNA staining showed an abnormal appearance with chromosomes widely dispersed in cells (Figure 2D). Prevention of bipolar spindle formation and accumulation of cyclin B1 were also seen with siRNA 183 or siRNA 1418 (data not shown). These results indicated that PLK-1 siRNA specifically reduced PLK-1 expression and blocked PLK-1 function.

Growth inhibition and induction of apoptosis by siRNA 1412 in vitro. We examined the effect of reduced PLK-1 expression for 72 hours following siRNA treatment. A modified 3-(4,5-dimethylthiazol-2-yl)-2,5-diphenyltetrazolium bromide (MTT) assay demonstrated a dose-dependent growth inhibition of bladder cancer cells. The $\mathrm{IC}_{50} \mathrm{~s}$ for the bladder cancer cell lines RT112, 253J, J82, SCaBER, TCCSUP, UM-UC-3, 5637, and RT4 were $35.1 \mathrm{nM}, 94.5 \mathrm{nM}, 119.3 \mathrm{nM}, 60.4 \mathrm{nM}, 73.0 \mathrm{nM}, 46.1 \mathrm{nM}, 40.0$ $\mathrm{nM}$, and $65.1 \mathrm{nM}$, respectively. These concentrations could reasonably be attained in vivo, especially using local administration to the bladder.

We examined the effect of the siRNA on the cell cycle of bladder cancer cells (UM-UC-3). The percentage of cells in $\mathrm{G}_{1} / \mathrm{S}$ phase decreased and the percentage of cells in $G_{2} / M$ phase increased after exposure to siRNA 1412 (100 nM) for 24 hours (Figure 3A). Moreover, increased numbers of sub- $G_{1}$ cells, which were expected to undergo apoptosis, were found 48 hours after exposure. In order to confirm induction of apoptosis, we investigated annexin V staining on siRNA-treated UM-UC-3 cells. As compared with untreated cells or control siRNA-treated cells, an abundance of siRNA 1412-treated cells were shown to be annexin V-positive apoptotic cells (Figure 3B). These results indicated that reduced expression of PLK-1 gave rise to growth inhibition and apoptosis induction in bladder cancer cells in vitro.

Before beginning in vivo trials, we confirmed that a pulse siRNA treatment could inhibit growth. Relatively high concentrations of siRNA $(100 \mathrm{nM})$ successfully prevented the proliferation of cancer cells even when applied for as little as 1 or 3 hours. Relative cell viabilities, compared with control cells, of UM-UC-3, 253J, and KU-7 cells treated with PLK-1 siRNAs were $0.187 \pm 0.0191$, $0.705 \pm 0.0342$, and $0.466 \pm 0.108$, respectively, after 1 hour of treat- 


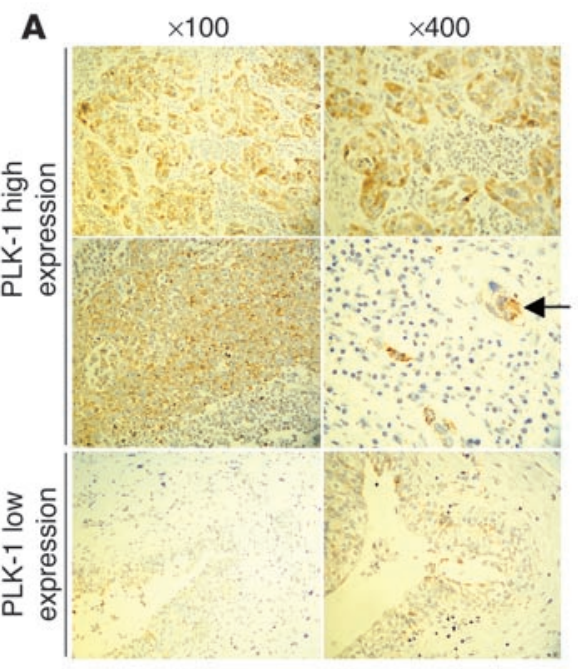

\section{Figure 1}

PLK-1 expression correlates with the progression of bladder cancer. (A) PLK-1 staining in the tissue of bladder cancers. Strong immune reaction in high-grade bladder cancers (top and middle rows) and weak immune reaction in low-grade bladder cancers (bottom row) were typically observed by immunohistochemistry. Nuclear staining was performed with Mayer's hematoxylin. The arrow indicates lymphatic invasion of cancer cells in the high-grade bladder cancer. Numbers indicate original magnifications. (B) Comparison of the survival curves between bladder cancers with high and those with low PLK-1 expression. (C) Western blotting analysis of PLK-1 expression in the tissues of bladder tumor (T) and normal bladder epithelium (N) of the same patients. (D) Western blotting analysis of PLK-1 expression in human bladder cancer and normal cells.

ment, and $0.0891 \pm 0.0290,0.399 \pm 0.0693$, and $0.243 \pm 0.0717$, respectively, after 3 hours of treatment (Figure 3C).

In vivo growth inbibition of bladder cancer in orthotopic murine models by transurethral administration of PLK-1 siRNA 1412. We established orthotopic bladder cancer mouse models. Among the bladder cancer cell lines we tested $(5637,253 \mathrm{~J}$, TCCSUP, and UM-UC-3), UMUC-3 was the only cell line that was orthotopically transplanted successfully. Shortly after implantation of UM-UC-3 $3^{\text {LUC }}$ cells into the bladder, high bioluminescence was demonstrated in all of the mice. Bioluminescence was not detectable the following day but was detected 1 week later and then increased substantially up to 4 weeks (Figure 4A). Before we examined the anticancerous effect of PLK-1 siRNA, we determined whether the cationic liposomes were able to deliver FITC-labeled siRNA to the orthotopically transplanted cancer cells. Abundant fluorescence was demonstrated in bladder cancer tissues when the siRNA/liposome complex was administered (Figure 4B). In contrast, no fluorescence could be seen when the siRNA alone was administered. Next, we examined

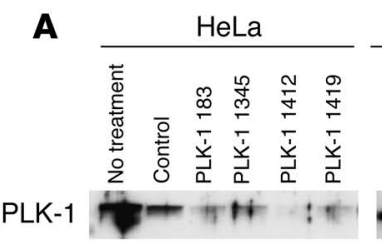

B

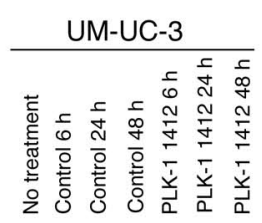

PLK-1 $\beta$-Actin
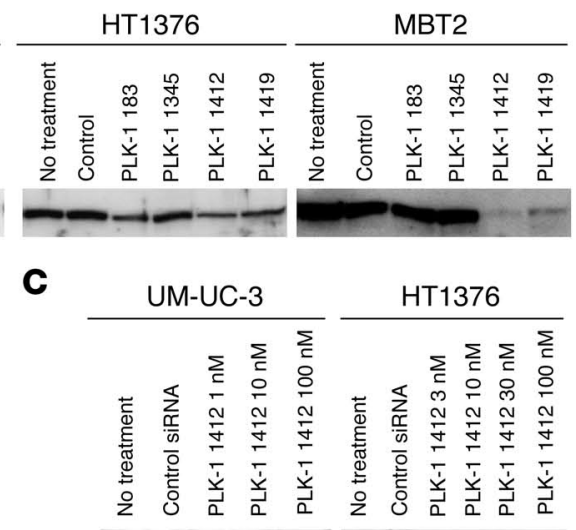

PLK-1

Cyclin B1

B-Actin
。
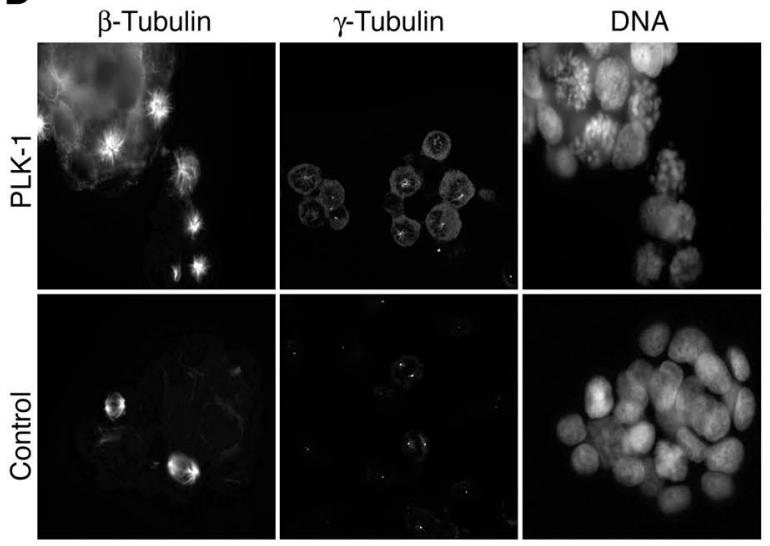

Figure 2

Prevention of PLK-1 expression and function by siRNA/cationic liposome complexes. (A) Comparison of the reduction of PLK-1 expression by 4 siRNAs. (B) Time-dependent inhibition of PLK-1 expression by PLK-1 siRNA 1412. (C) Dose-dependent inhibition of PLK-1 expression and accumulation of cyclin B1 by PLK-1 siRNA 1412. (D) Prevention of bipolar spindle formation by PLK-1 siRNA (100 nM, 24 hours). $\beta$-Tubulin staining, $\gamma$-tubulin staining, and Hoechst 33342 DNA staining of bladder cancer cells (UM-UC-3) are shown. 
A

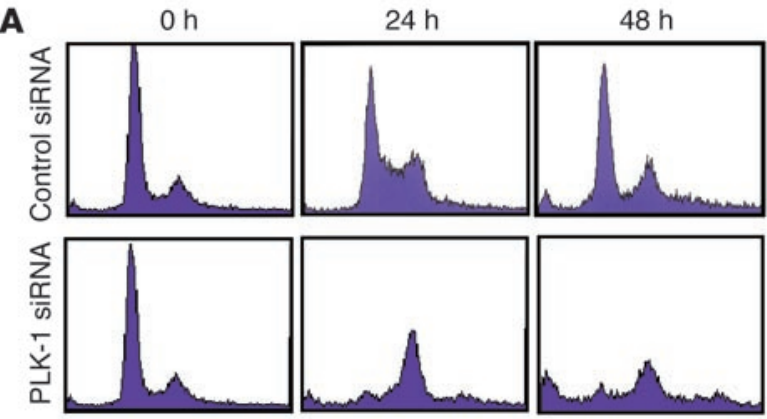

B No treatment
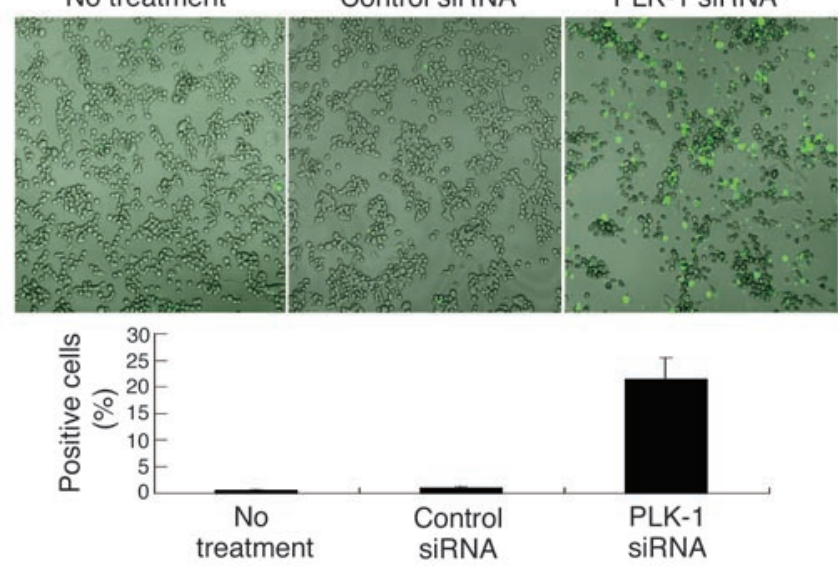

C

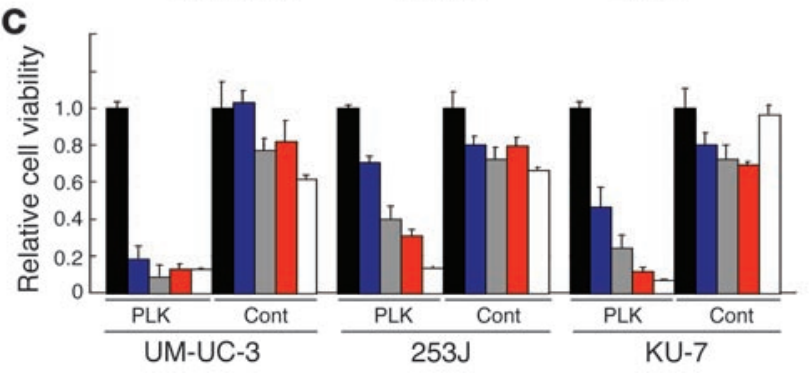

whether the siRNA/cationic liposome complex reduced PLK-1 expression of the orthotopically transplanted cancer cells. Abundant expression of PLK-1 was demonstrated in bladder cancer tissues of control mice (Figure 4C); in contrast, only weak expression was observed in PLK-1 siRNA/liposome-treated mice. These results suggested that the intravesical siRNA/liposome complex could successfully insert into bladder tissues and would be able to work as an anticancer agent.

We investigated the growth-inhibitory effect of the complex of PLK-1 siRNA and liposomes in vivo. Figure 4D shows the growth curves of the respective murine transplanted cancers. Although equivalent numbers of cancer cells were injected, cancer growth rates differed among the treatment groups. Photon emissions of mice treated with both concentrations $(600 \mathrm{nM}$ and $6 \mu \mathrm{M})$ of PLK-1 siRNA were significantly lower than those of mice from the untreated or the control siRNA group $(P<0.05)$.

We surveyed 20 sections (taken at $40-\mu \mathrm{m}$ intervals) from each murine bladder in order to examine whether bladder cancers existed in the mice. Bladder cancers were apparent in all of the mice in which we observed high bioluminescence with an in vivo imaging system (IVIS; Xenogen Corp.) (Figure 4E, top left and

\section{Figure 3}

Growth inhibition and induction of apoptosis in bladder cancer cells by PLK-1 depletion using PLK-1 siRNA 1412 with cationic liposomes. (A) Cell cycle alteration of bladder cancer cells by PLK-1 siRNA 1412 (100 nM, 24 and 48 hours) was demonstrated by FACS analysis using propidium iodide staining. (B) Apoptosis induction by PLK-1 siRNA ( $100 \mathrm{nM}, 36$ hours) was identified by FITC-annexin V staining. Original magnification, $\times 100$. Histogram shows the results from 3 independent experiments (500 cells each). Error bars indicate SD. (C) Comparison of growth inhibition by PLK-1 siRNA with different treatment times. PLK, cells treated by PLK-1 siRNA (100 nM); Cont, cells treated by control siRNA (100 nM). Relative cell growth was measured 72 hours after the beginning of treatments. Error bars indicate SD. Black bars, no treatment; blue bars, treated for 1 hour; gray bars, treated for 3 hours; red bars, treated for 6 hours; white bars, treated for 72 hours.

middle). However, we could not detect any cancer cells in 2 of the animals treated with $6 \mu \mathrm{M}$ PLK- 1 siRNA and 1 of the animals treated with 600 nM PLK-1 siRNA; we were also unable to detect bioluminescence by IVIS in these animals (Figure 4E, top right). The mucosal surfaces of these PLK-1 siRNA-treated murine bladders did not show apparent injury (Figure 4E, bottom), and there were no differences microscopically in the noncancerous bladder mucosa among the PLK-1 siRNA-treated, control siRNA-treated, and untreated mice. We found no differences in the body weight and laboratory examination of peripheral blood among these murine groups. These results indicated that PLK-1 siRNA 1412 inhibited the growth of bladder cancers not only in vitro, but also in vivo, and some of the mice showed a complete eradication of the cancer cells without any severe adverse effects. These data suggest that PLK-1 siRNA 1412 will be an attractive therapeutic tool for bladder instillation therapy.

Recently, siRNA has been reported to activate IFN signaling, inducing an immune response (14). In order to examine the role of immune responses in the in vivo growth-inhibitory effect, we investigated the transcription levels of IFN- $\alpha,-\beta$, and $-\gamma$ genes in murine bladder tissues. However, neither PLK-1 siRNA nor control siRNA induced the expression of these genes (data not shown). Since we may have been unable to detect the changes in IFN levels in vivo, we investigated IFN induction by these siRNAs in the murine monocyte cell line RAW264.7 in vitro. The siRNAs did not induce transcription of IFN- $\beta$ gene in RAW264.7 cells (Figure 4F). These results indicated that the in vivo growth-inhibitory effect demonstrated by the complex of PLK-1 siRNA and liposome was caused primarily by RNA interference, not by innate immunity.

\section{Discussion}

Intravesical BCG, which is currently the most effective agent for bladder cancer treatment, causes irritative voiding symptoms in $90 \%$ of cases because of the inflammation that may be necessary for the effective action of this agent. Moreover, BCG sepsis, although rare (occurring in $0-4 \%$ of patients who underwent intravesical BCG therapy), is a life-threatening condition. Therefore, options of intravesical therapy are needed. RNA interference mediated by siRNA is used as a sequence-specific gene-silencing mechanism and currently has become an indispensable tool in the study of the regulation of gene expression and function in vitro $(4,5)$. siRNA is a potential therapeutic agent based on rational gene-based drug design. In vivo, however, reports that have demonstrated successful treatment using siRNAs are scarce (15-17). 
Establishment of a functional DDS is still crucial for development of therapeutically viable nucleic acid-related medicines. We focused on bladder cancer since it grows in a closed cavity, the bladder. In this environment, local administration of the complex of siRNA and cationic liposomes could provide a high concentration of the agent to attach to the target cancer cells during a limited time. The cationic liposome LIC101, whose safety has already been verified by systemic i.v. injection into animals (13), was used as a carrier in this study. LIC101 has a low cytotoxicity and high efficacy of transfection. Intravesical siRNA is a novel strategy whose efficacy, to our knowledge, has never been demonstrated before our study. In this study, we demonstrated that PLK-1 siRNA 1412 in cationic liposome was transfected into bladder cancer cells in the murine orthotopic bladder cancer model. These cells were monitored frequently, quantitatively, and noninvasively in real time by IVIS (Figure 4A), and we demonstrated that intravesical PLK-1 siRNA successfully inserted into cells (Figure 4B), reduced PLK-1 expression (Figure 4C), and prevented growth of the bladder cancers (Figure 4D).

With regard to siRNA therapy, there are some beneficial points in intravesical administration. In view of the cost performance, local administration of siRNA is superior to i.v. injection. In vivo gene silencing by general administration of siRNA/liposome complexes has been reported $(18,19)$. However, intravesical local administration seemed to achieve similar effects with a 50- to 100-fold lower dose of the complex, although the targeted genes were different. The procedure of transurethral administration is quite simple and repeatable in clinic, although administration into murine bladder is relatively difficult. The estimated side effect of intravesical administration would be less than that of general administration.
A Time after transplantation
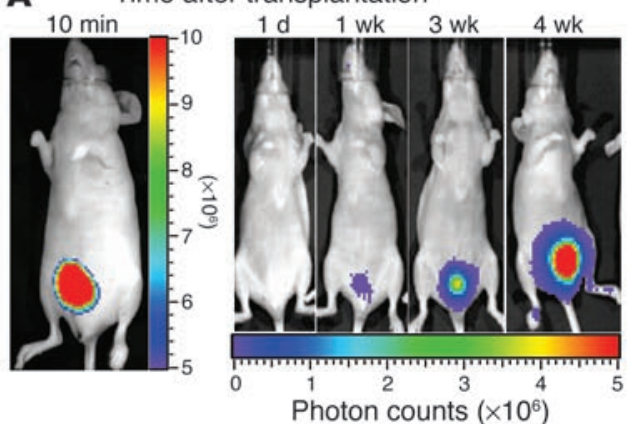

D
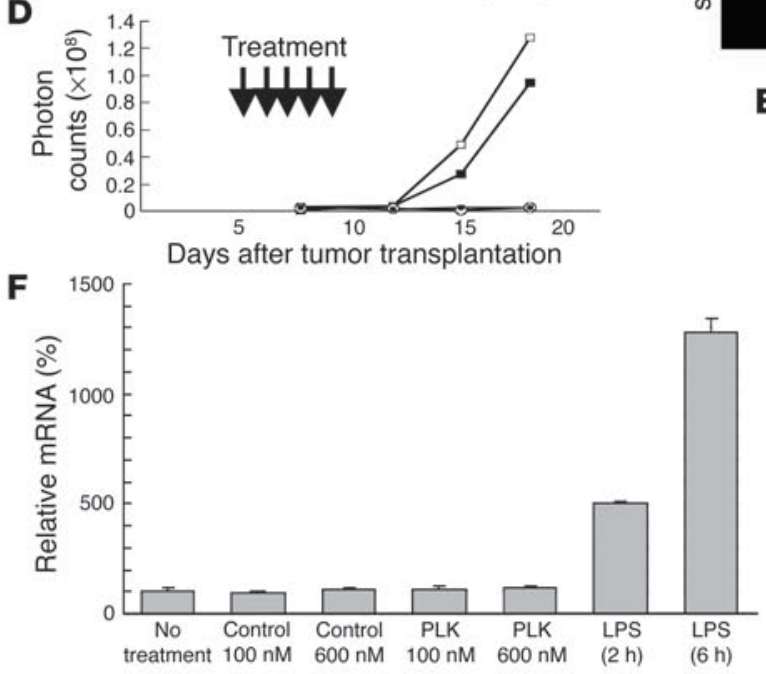
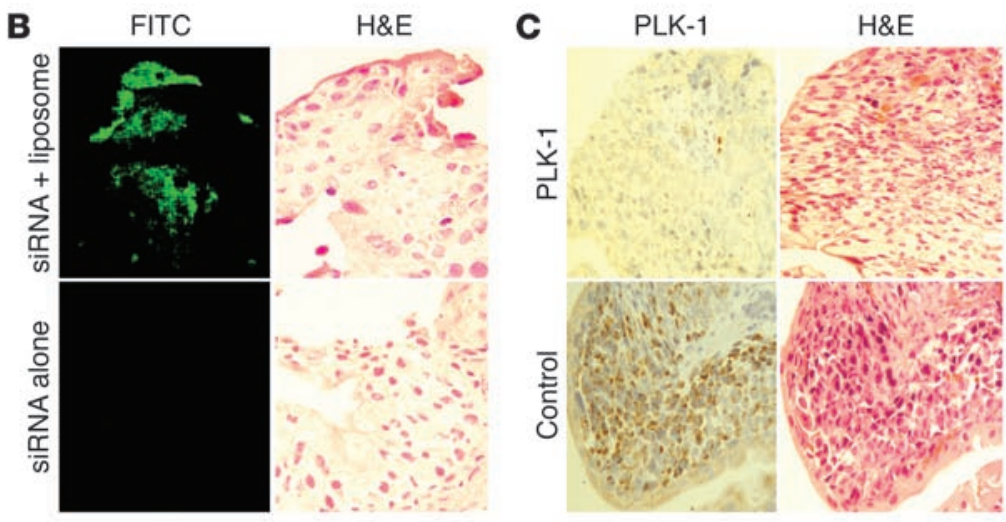

E
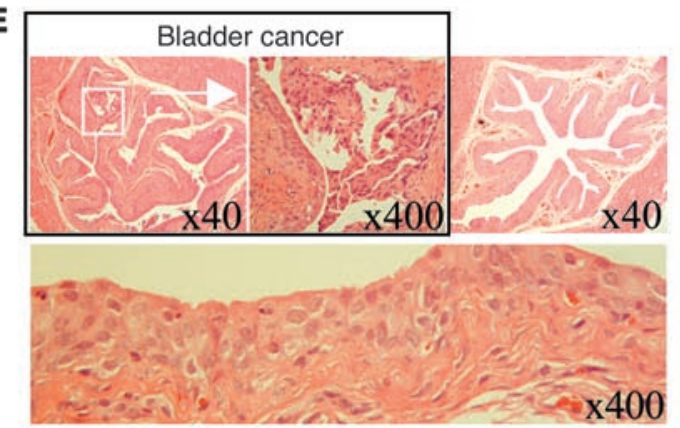

Normal bladder mucosa

Figure 4

Growth inhibition of bladder cancer by PLK-1 siRNA in orthotopic bladder cancer mouse models. (A) Images were obtained by IVIS at 10 minutes, 1 day, 1 week, 3 weeks, and 4 weeks after transplantation. The photon counts of each mouse are indicated by the pseudo-color scales. (B) FITC-labeled control siRNA with cationic liposomes was successfully transfected into bladder cancer cells as shown by immune staining using an anti-FITC antibody (top left panel). H\&E counterstaining was performed to identify cancer cells (top right panel). The same experiments were performed without cationic liposomes (bottom panels). Original magnification, $\times 400$. (C) Immunohistochemical staining revealed that intravesical PLK-1 siRNA/liposome complexes successfully reduced PLK-1 expression (top left panel). H\&E counterstaining was performed to identify cancer cells (top right panel). The same experiments were performed with the complex of control siRNA and liposomes (bottom panels). Original magnification, $\times 400$. (D) The growth curves of orthotopically transplanted UM-UC-3 $3^{\text {LUC }}$ cells were measured by IVIS. The anticancerous effect of intravesical PLK-1 siRNA was demonstrated in vivo. Open squares, no treatment; filled squares, treatment with control siRNA $(6 \mu \mathrm{M})$; open circles, treatment with PLK-1 siRNA $(6 \mu \mathrm{M})$; filled circles, treatment with PLK-1 siRNA (600 nM). The data shown are representative of duplicate experiments. (E) Pathological analysis was performed in UM-UC-3LUC_transplanted murine bladder tissues after PLK-1 siRNA treatment. Residual cancer (top left and middle panels) and noncancerous bladder (top right and lower panels) were observed microscopically. Numbers indicate original magnification. (F) No apparent induction of IFN- $\beta$ gene expression by either PLK-1 or control siRNAs was observed in RAW264.7 cells. 
We hope intravesical siRNA therapy will obtain an excellent anticancerous effect without adverse effects.

Among the many regulators that contribute to mitosis, PLK- 1 is thought to be critical and to mediate multiple mitotic processes, including activation of the CDC25C-Cdkl amplification loop, regulation of entry into mitosis, establishment of the bipolar spindle, regulation of the anaphase-promoting complex, segregation of chromosomes, and cytokinesis (6). PLK-1 is altered in various cancers, including breast cancer, non-small cell lung cancer, head and neck squamous cell cancer, and melanoma (6-10). We demonstrated here that the histological grade, deep tissue invasiveness, and lymphatic and venous invasiveness are positively correlated with PLK-1 expression, and bladder cancer tissues as well as cell lines demonstrated abundant expression of PLK-1 (Figure 1, A, $\mathrm{C}$, and D, and Table 1). Moreover, high expression of PLK-1 is suggested to be a predictor of poor prognosis (Figure 1B). PLK-1 depletion dramatically inhibited formation of the bipolar spindles (Figure 2D), induced cell cycle arrest (Figure 3A), prevented cell proliferation (Figure $3 \mathrm{C}$ ), and resulted in induction of apoptosis (Figure 3B), in bladder cancer cells. These data are consistent with the results from many different cancer cell types (12). Moreover, in our experiments PLK-1 siRNA 1412, which we first designed and which is identical to the mRNA sequence of murine plk-1, was more effective than the siRNAs used previously $(11,12)$.

In order to successfully deliver siRNAs in vivo, several intelligent methods have been reported, including use of cationic liposomes, viral vectors, and hydrodynamic delivery $(17,18,20-22)$. The largevolume hydrodynamic tail vein administration was shown to effectively deliver siRNA to liver and some other organs (20). However, for the present this method is not feasible for therapeutic approaches in humans, although progress in medical engineering may support it in the future. Viral vectors are the most efficient means to transfer therapeutic genes into many targets in vivo. The vector-based side effects, however, limit their value in clinical applications. Iatrogenic-induced leukemia as a consequence of insertional mutagenesis events warrants a thorough reassessment of the potential risk of virus vector-mediated gene therapy (22-24). Although vector-mediated mutagenesis was only reported in retrovirus vectors, other viral vectors might pose similar risks. Very recently, a sophisticated method of systemic application of a pBS/U6 plasmid vector containing a short hairpin RNA that targeted PLK-1 together with the nuclease inhibitor aurintricarboxylic acid was demonstrated to inhibit tumor growth in a murine subcutaneous tumor model (17).

In this study we used a cationic liposome as a carrier of siRNA. The transient effects of siRNA/cationic liposome complexes have the advantage of being easily controllable; the starting and end points of the therapy are easily modified. If an unexpected adverse side effect were seen, suspension of treatment and consequent diminishing of the siRNA effect would be prompt. Currently, Vitravene is the only antisense drug approved by the US FDA (25). This phosphorothioate oligonucleotide is intravitreally injected to treat CMV-induced retinitis in patients with AIDS. The local administration into the vitreal cavity can deliver a high concentration of the agent to the target virus. The discovery that short double-stranded RNA molecules can silence gene expression in mammalian cells has been a significant breakthrough in nucleic acid biology, and this method is significantly more efficient than traditional antisense approaches $(4,5)$, although siRNA still has the barrier in DDS. Our data indicate that intravesical siRNA is a viable alternative to overcome DDS problems.
In conclusion, we have demonstrated that intravesical administration of PLK-1-targeted siRNA/cationic liposomes inhibited cancer growth in murine orthotopic bladder cancer models. The in vivo growth-inhibitory effect demonstrated by the complex of PLK-1 siRNA and liposome was caused primarily by RNA interference, not by innate immunity. This is the first demonstration, to our knowledge, of inhibition of cancer growth in the murine bladder by intravesical siRNA/cationic liposomes. We believe that transurethral siRNA therapy can overcome the DDS problem of siRNA delivery and cause a breakthrough in clinical applications of nucleic acid therapeutics. The efficacy and safety of intravesical siRNA should be verified by early-phase clinical trials.

\section{Methods}

Patients and human samples. All 58 patients underwent radical cystectomy and simultaneous bilateral pelvic lymph node dissection at the Department of Urology, Shiga University of Medical Science, between 1979 and 1995. Samples of fresh tumor and normal epithelium were stored at $-80^{\circ} \mathrm{C}$, and parallel samples were fixed in formalin, embedded in paraffin, and sectioned for use in microscopic analysis. Informed consent was provided according to the World Medical Association Declaration of Helsinki. Clinical and pathological data were obtained by retrospective chart review, as previously described (26).

Animals, cell lines, and reagents. Approval for these studies was obtained from the Committee on Animal Research of the Kyoto University Faculty of Medicine. Specific pathogen-free 6- to 8-week-old BALB/c nu/nu mice were used (Japan SLC Inc.). The human bladder cancer cell lines 253J, 5637, HT1197, HT1376, J82, RT4, RT112, SCaBER, TCCSUP, KU-7, and UM-UC-3; the mouse bladder cancer cell line MBT2; human primary hepatocytes; the normal human fibroblast cell lines HF and NHDF; and the murine monocyte cell line RAW264.7 were obtained from the American Type Culture Collection. Four PLK-1 (GenBank accession number NM_005030) siRNAs and a nonsense siRNA used as a control were chemically synthesized (Nippon Shinyaku Co.). The sequences of the sense strands were: siRNA 183, 5'-GGGCGGCUUUGCCAAGUGCdTdT-3'; siRNA 1345, 5'-GACAGCCUGCAGUACAUAGdTdT3'; siRNA 1412, 5'-CCUUGAUGAAGAAGAUCACdTdT-3'; siRNA 1418, 5'-TGAAGAAGAUCACCCUCCUdTdT-3'; and control, 5'-UUCUCCGAACGUGUCACGUdTdT-3'. The RNA sequences of siRNA 183 and siRNA 1418 are equivalent to those of the siRNAs used in previous studies (11, 12). Positively charged liposomes containing a cationic lipid analogue were synthesized at Nippon Shinyaku Co., as described previously (13).

Growth-inhibitory effects of PLK-1 siRNA in vitro. Cell proliferation was determined by the modified MTT assay with SF reagent (Nacalai Tesque Inc.), as described previously (15). The mean of 5 samples was calculated. $\mathrm{IC}_{50}$ values were obtained using the nonlinear regression program CalcuSyn (Biosoft).

Cell cycle analysis. Cell cycle analysis using propidium iodide and fluorescence-activated cell sorting (FACS; BD Biosciences) was performed as described previously (27).

Western blotting analysis. Western blotting analyses were performed as described previously (28). Rabbit polyclonal anti-PLK-1 antibody (Upstate Biotechnology Inc.), rabbit polyclonal anti-cyclin B1 antibody (Santa Cruz Biotechnology Inc.), and rabbit polyclonal anti-actin antibody (SigmaAldrich) were used as primary antibodies.

Immunocytochemistry. Immunocytochemistry using $\beta$-tubulin-specific and $\gamma$-tubulin-specific antibodies (Sigma-Aldrich) and Hoechst 33342 DNA staining (Invitrogen Corp.) was performed according to the manufacturers' protocol. To detect apoptotic cells, we performed annexin $V$ staining using the MEBCYTO Apoptosis Kit (Medical and Biological Laboratories Co.). 
Immunobistochemical staining. Immunohistochemical staining was performed by the conventional avidin-biotin-peroxidase complex method (VECTASTAIN Elite ABC kit; Vector Laboratories Inc.), as described previously (26). Anti-human PLK-1 mAb (Transduction Laboratories) with a 1:100 dilution was used as the primary antibody. Sections were counterstained with hematoxylin and mounted. Normal mouse IgG was used instead of primary antibody as a negative control.

Immunohistochemical evaluation. Two independent investigators ( $T$. Yuasa and Y. Toda) calculated the number of PLK-1-positive tumor cells in a minimum of 15 fields. Staining results were assessed in a semiquantitative fashion by the 2 investigators independently. Prognostic evaluation was based on the mean PLK-1 index (percent PLK-1-positive cells). This categorization is fundamentally similar to that of previous immunohistochemical studies $(5,29)$.

Real-time quantitative RT-PCR. Mice were treated, by intravesical administration, with $100 \mu \mathrm{l}$ of the complex of liposome and either PLK-1 siRNA $(6 \mu \mathrm{M}$ or $600 \mathrm{nM})$ or control siRNA $(6 \mu \mathrm{M})$, and LPS $(100 \mu \mathrm{g} / \mathrm{ml})$. Six hours after the treatment, the bladder tissues were removed. In vitro, RAW264.7 mouse monocyte-derived cells were cultured with or without the same siRNA complexes for 6 hours. Total RNA $(1 \mu \mathrm{g})$ was converted to $50 \mu \mathrm{l} \mathrm{cDNA}$, and the transcriptional levels of murine IFNs ifn $\alpha$, ifn $\beta$, and if $n \gamma$ were analyzed using the LightCycler System (Roche Diagnostics Corp.) with FastStart DNA Master SYBR Green I (Roche Diagnostics Corp.), as described previously (30).

Generation of stable LUC-expressing cancer cell lines. Tumor cell lines were stably transfected with the pGL3-control vector (Promega Corp.) and with pSV2Neo (American Type Culture Collection) using Lipofectamine 2000 (Invitrogen Corp.), as described previously (31).

Mouse models. In order to establish the orthotopic bladder cancer models, LUC-labeled bladder cancer cells $\left(2 \times 10^{6}\right)$ were implanted into the murine bladder cavity via 24-gauge angiocatheters (Surflo; Terumo Corp.), as described previously (32).

In vivo effects of PLK-1 siRNA. Mice were administered the LUC-expressing tumor cells on day 0 . On day 4 , each mouse was observed by IVIS (Xenogen Corp.), and bioluminescence from implanted cancer cells was measured. These mice were divided into 4 treatment groups with equivalent levels of bioluminescence among the groups. Transurethral treatment with siRNAs was performed 5 times, once on each of days 5-9. Mice were anesthetized with an i.p. injection of Nembutal $(75 \mu \mathrm{g} / \mathrm{kg})$. The bladders were catheterized and washed with PBS, and siRNA/liposomes were administered, followed by a purse-string suture placed around the urethra to occlude it. After 4 hours, the sutures were removed and the mice restarted their spontaneous voiding. Mice were divided into 4 groups: (a) untreated mice; (b) mice treated with $6 \mu \mathrm{M}$ control siRNA; (c) mice treated with $600 \mathrm{nM}$ PLK-1 siRNA 1412; and (d) mice treated with $6 \mu \mathrm{M}$ PLK-1 siRNA 1412. Each group contained 7 mice, and these treatment regimes were performed twice. The in vivo growth of cancer cells was monitored by IVIS, as described previously (31). On day 21, all mice were killed humanely, and their blood was collected and analyzed for cell counts of red blood cells, white blood cells, and platelets, and serum concentrations of aspartate aminotransferase, alanine aminotransferase, lactate dehydrogenase, total protein, creatinine, and blood urea nitrogen.

Statistical analysis. The relationship between the staining levels (PLK-1 index) and various clinicopathological factors was analyzed using the $\chi^{2}$ or the Kruskal-Wallis test. Kaplan-Meier analysis was used to estimate the cumulative cause-specific survival rate, and the log-rank test was used to correlate differences in patient survival with staining intensity of PLK-1. The influence of siRNA on the growth of bladder cancers was analyzed by the Student's $t$ test. We used StatMate III (ATMS Co.) statistical software. In all statistical analyses, $P$ values of 0.05 or less were judged to be statistically significant.

\section{Acknowledgments}

This work was partly supported by the Uehara Memorial Foundation, the Foundation for Promotion of Cancer Research, the Public Trust Haraguchi Memorial Cancer Research Fund, the Ichiro Kanehara Foundation, the Fujiwara Memorial Foundation, Grants-in-Aid for Scientific Research from the Ministry of Education, Culture, Sports, Science and Technology of Japan, and the Center of Excellence program of the Ministry of Education, Culture, Sports, Science and Technology of Japan.

Received for publication August 16, 2004, and accepted in revised form January 18, 2005.

Address correspondence to: Takeshi Yuasa, Department of Transfusion Medicine and Cell Therapy, Kyoto University Hospital, 54 Shogoin-Kawahara, Sakyo-ku, Kyoto 606-8507, Japan. Phone: 81-75751-3630; Fax: 81-75-751-3631; E-mail: yuasa@kuhp.kyoto-u.ac.jp.

Masaki Nogawa's present address is: Discovery Research Laboratories, Nippon Shinyaku Co. Ltd., Kyoto, Japan.
1. Messing, E.M. 2001. Urothelial tumors of the urinary tract. In Campbell's urology. P.C. Walsh, A.B. Retik, E.D. Vaughan Jr., and A.J. Wein, editors. W.B. Saunders Co. New York, New York, USA. 2732-2784.

2. Gee, J., Sabichi, A.L., and Grossman, H.B. 2002. Chemoprevention of superficial bladder cancer. Crit. Rev. Oncol. Hematol. 43:277-286.

3. Cookson, M.S., et al. 1997. The treated natural history of high risk superficial bladder cancer: 15 -year outcome. J. Urol. 158:62-67.

4. Lieberman, J., Song, E., Lee, S.K., and Shankar, P. 2003. Interfering with disease: opportunities and roadblocks to harnessing RNA interference. Trends Mol. Med. 9:397-403.

5. Dorsett, Y., and Tuschl, T. 2004. siRNAs: applications in functional genomics and potential as therapeutics. Nat. Rev. Drug Discov. 3:318-329.

6. Barr, F.A., Sillje, H.H., and Nigg, E.A. 2004. Pololike kinases and the orchestration of cell division. Nat. Rev. Mol. Cell Biol. 5:429-440.

7. Holtrich, U., et al. 1994. Induction and down-regulation of PLK, a human serine/threonine kinase expressed in proliferating cells and tumors. Proc.
Natl. Acad. Sci. U. S. A. 91:1736-1740.

8. Wolf, G., et al. 1997. Prognostic significance of polo-like kinase (PLK) expression in non-small cell lung cancer. Oncogene. 14:543-549.

9. Strebhardt, K., Kneisel, L., Linhart, C., Bernd, A., and Kaufmann, R. 2000. Prognostic value of polo-like kinase expression in melanomas. JAMA. 283:479-480

10. Knecht, R., et al. 1999. Prognostic significance of polo-like kinase (PLK) expression in squamous cell carcinomas of the head and neck. Cancer Res. 59:2794-2797.

11. Spankuch-Schmitt, B., Bereiter-Hahn, J., Kaufmann, M., and Strebhardt, K. 2002. Effect of RNA silencing of polo-like kinase-1 (PLK1) on apoptosis and spindle formation in human cancer cells. J. Natl. Cancer Inst. 94:1863-1877.

12. Liu, X., and Erikson, R.L. 2003. Polo-like kinase (Plk) 1 depletion induces apoptosis in cancer cells. Proc. Natl. Acad. Sci. U. S. A. 100:5789-5794.

13. Hirabayashi, K., et al. 1999. Inhibition of cancer cell growth by polyinosinic-polycytidylic acid/cationic liposome complex: a new biological activity. Cancer
Res. 59:4325-4333.

14. Sledz, C.A., Holko, M., de Veer, M.J., Silverman, R.H., and Williams, B.R. 2003. Activation of the interferon system by short-interfering RNAs. Nat. Cell Biol. 5:834-849.

15. Brummelkamp, T.R., Bernards, R., and Agami, R. 2002. Stable suppression of tumorigenicity by virus-mediated RNA interference. Cancer Cell. 2:243-247.

16. Song, E., et al. 2003. RNA interference targeting Fas protects mice from fulminant hepatitis. Nat. Med. 9:347-351.

17. Spankuch, B., et al. 2004. Cancer inhibition in nude mice after systemic application of U6 promoter-driven short hairpin RNAs against PLK1. J. Natl. Cancer Inst. 96:862-872.

18. Sioud, M., and Sorensen, D.R. 2003. Cationic liposome-mediated delivery of siRNAs in adult mice. Biochem. Biophys. Res. Commun. 312:1220-1225.

19. Sorensen, D.R., Leirdal, M., and Sioud, M. 2003. Gene silencing by systemic delivery of synthetic siRNAs in adult mice. J. Mol. Biol. 327:761-766.

20. Lewis, D.L., Hagstrom, J.E., Loomis, A.G., Wolff, 
J.A., and Herweijer, H. 2002. Efficient delivery of siRNA for inhibition of gene expression in postnatal mice. Nat. Genet. 32:107-108.

21. Zender, L., et al. 2003. Caspase 8 small interfering RNA prevents acute liver failure in mice. Proc. Natl. Acad. Sci.U. S. A. 100:7797-7802.

22. Hacein-Bey-Abina, S., et al. 2002. Sustained correction of X-linked severe combined immunodeficiency by ex vivo gene therapy. N. Engl. J. Med. 346:1185-1193.

23. McCormack, M.P., and Rabbitts, T.H. 2004. Activation of the T-cell oncogene LMO2 after gene therapy for X-linked severe combined immunodeficiency. N. Engl.J. Med. 350:913-922.

24. Hacein-Bey-Abina, S., et al. 2003. A serious adverse event after successful gene therapy for X-linked severe combined immunodeficiency. N. Engl.J. Med. 348:255-256.

25. Marwick, C. 1998. First "antisense" drug will treat CMV retinitis. JAMA. 280:871.

26. Kageyama, S. 2002. High expression of human uroplakin Ia in urinary bladder transitional cell carcinoma. Jpn.J. Cancer Res. 93:523-531.

27. Kimura, S., Maekawa, T., Hirakawa, K., Murakami, A., and Abe, T. 1995. Alterations of c-myc expression by antisense oligodeoxynucleotides enhance the induction of apoptosis in HL-60 cells. Cancer Res. 55:1379-1384

28. Kuroda, J., et al. 2003. The third-generation bisphosphonate zoledronate synergistically augments the anti-Ph+ leukemia activity of imatinib mesylate. Blood. 102:2229-2235.
29. Knecht, R., Oberhauser, C., and Strebhardt, K. 2000. PLK (polo-like kinase), a new prognostic marker for oropharyngeal carcinomas. Int. J. Cancer. 89:535-536.

30. Luo, J.L., Maeda, S., Hsu, L.C., Yagita, H., and Karin, M. 2004. Inhibition of NF-kappaB in cancer cells converts inflammation-induced tumor growth mediated by TNFalpha to TRAIL-mediated tumor regression. Cancer Cell. 6:297-305.

31. Nogawa, M., et al. 2005. Monitoring luciferaselabeled cancer cell growth and metastasis in different in vivo models. Cancer Lett. 217:243-253.

32. Tanaka, M., et al. 2003. Noninvasive detection of bladder cancer in an orthotopic murine model with green fluorescence protein cytology. J. Urol. 170:975-978. 